\title{
Lung weights and undiagnosed lung lesions in medico-legal autopsies in Dar es Salaam, Tanzania
}

william mawalla ( $\square$ mawallawf@ymail.com)

Muhimbili University of Health and Allied Sciences School of Medicine https://orcid.org/0000-00020016-7551

\section{Henry A. Mwakyoma}

Muhimbili University of Health and Allied Sciences

Upendo Massamu

Muhimbili University of Health and Allied Sciences

\section{Research article}

Keywords: lung lesions, medico-legal, autopsy

Posted Date: June 22nd, 2019

DOI: https://doi.org/10.21203/rs.2.10569/v1

License: (c) (i) This work is licensed under a Creative Commons Attribution 4.0 International License.

Read Full License

Version of Record: A version of this preprint was published at Forensic Science International: Reports on December 1st, 2020. See the published version at https://doi.org/10.1016/j.fsir.2020.100120. 


\section{Abstract}

Background Medico-legal or forensic autopsy is a special type of autopsy performed under the orders of a legal authority in circumstances involving unnatural, suspicious or criminal deaths. Gross and histopathological studies of viscera in medico-legal autopsies have been observed to serve as crucial tool in the detection of undiagnosed disease conditions and the resulting findings may closely represent the general population. Methods A prospective cross sectional study was carried out at the Muhimbili National Hospital $(\mathrm{MNH})$ department of pathology, to medico-legal autopsies received at the $\mathrm{MNH}$ mortuary. The study included 103 cases in an eight month period. Both lungs were weighed for each case and tissue samples were collected from each lobe, in both lungs. Other gross findings were observed and recorded. Tissue sections were made and stained with hematoxylin and eosin and evaluated under a microscope. Results Out of 103 cases studied, $88.3 \%$ were male with mean age of $35.8+16.6$ years. In females, the mean age was $31.6+10.4$ years. The mean weight of lungs was found to be $627.3+138.5 \mathrm{~g}$ and $591.7+129.7 \mathrm{~g}$ for the right and left lung, respectively, in males. In females, the mean weight of lungs was $616.7+166.1 \mathrm{~g}$ and $583.3+155.8 \mathrm{~g}$ for the right and left lung, respectively. More than half $(53.4 \%)$ of lung samples had some pathological disease, and the leading diagnosis was pulmonary congestion/oedema (22.3\%) followed by lobar pneumonia (18.5\%). Age was found to relate with the observed histological diagnosis of the lungs. Conclusion Males, especially the youth and adults are the main victims of medico-legal deaths. The study also revealed high prevalence of undiagnosed lung diseases in victims of medico-legal deaths. These findings may reflect heavy burden of lung diseases in the general population.

\section{Background}

Autopsy is the process of systematically examining a dead body with objectives of identifying the primary cause of death ${ }^{[1]}$. Medico-legal or forensic autopsy is performed under the orders of a legal authority in circumstances relating to unnatural, sudden, suspicious or criminal deaths. The autopsy findings and underlying cause of death can then be utilized for legal purposes to assist the course of justice. Qualified forensic pathologists are the ones entitled to perform and give findings for medico-legal autopsies ${ }^{[2,3]}$. Gross and histopathological studies of viscera in medico-legal autopsies have been observed to not only aid in ascertaining the cause of death but also serve as crucial tool in detection of

undiagnosed disease conditions, particularly, lung diseases ${ }^{[4]}$. Furthermore, studies ${ }^{[5]}$ in various settings reveal that a significant percentage of autopsies demonstrate findings that were not previously clinically diagnosed.

Chronic respiratory diseases (CRD) affect hundreds of millions of people of all ages across the globe. Majority of the affected population lives in low- or middle-income countries or deprived populations ${ }^{[6,7]}$. Primary respiratory infections, such as bronchitis, bronchopneumonia and other forms of pneumonia are common in clinical and pathological practice. Rising global air pollution with environmental and occupational inhalants and chemical toxic substances render chronic lung diseases such as; chronic 
bronchitis and pulmonary edema, fractious. Also, lungs have been found to be secondary target most terminal diseases; so that some degree of pulmonary edema, atelectasis, or bronchopneumonia is present in virtually every terminally-ill patient ${ }^{[4]}$.

Establishing normal organ weights, especially of the lungs is important, as it can hint pathological changes occurring in organs besides elucidating the relationship of trauma and disease. Various races and regions of the world will have different organ weights.

Organ weights in 684 Caucasoid adults who died of external causes with no pathological finding revealed mean lung weight in males to be $663+239 \mathrm{~g}$ and $583+216 \mathrm{~g}$ for the right and left lung, respectively. In females, the weight was $546+207 \mathrm{~g}$ and $467+174 \mathrm{~g}$ for the right and left lung, respectively. ${ }^{[8]}$

A study ${ }^{[5]}$ on normal organ weights of Indian adults(18-75 years) done in India showed the postmortem average weight of non-pathological lungs in males was between; 410 to $510 \mathrm{~g}$ and 370 to $470 \mathrm{~g}$ in the right and left lung, respectively. In female, the weight was 360 to $460 \mathrm{~g}$ and 360 to $460 \mathrm{~g}$ in right and left lung, respectively. On the other hand, a case study done involving medico-legal autopsy cases in Zambia showed; weight of the lungs to be $442.0 \pm 152.3 \mathrm{~g}$ left lung, and $504.6 \pm 174.6 \mathrm{~g}$ right lung in the male population. In the female population the weight of the left lung was $365.7 \pm 119.8 \mathrm{~g}$ and the right lung $405.0 \pm 116.0$ g. $^{[9]}$

With undiagnosed lung lesions, a study by Jhajj etal ${ }^{[10]}$ of histopathological examination in medico-legal autopsies in Faridkot, Punjab has revealed the most common respiratory system findings to be pneumonia followed by tuberculosis, pulmonary candidiasis and carcinoma of the lung. In contrary, a histopathological study by Selvam etal ${ }^{[11]}$ in Salem district, India showed that pulmonary tuberculosis was the most common finding followed by pulmonary eodema and then pneumonitis. Another study on the histopathological examination in medico-legal autopsy in India ${ }^{[12]}$ showed higher case incidence in third and fourth decades of life. Males were more prone to death by diseases (63.33\%) as compared to females $(36.67 \%)$ with the respiratory system involved in $30 \%$ of the cases. Lungs oedema was noticed during autopsy in $37.78 \%$ cases and confirmed by histopathology in $33 \%$ cases. Pneumonic changes were observed in lungs during autopsy in $31.11 \%$ cases while confirmed by histopathology in only $24.44 \%$ cases. Tubercular changes in lungs were noticed in $8.89 \%$ cases and confirmed by histopathology in $13.33 \%$ cases.

In Africa, a review of autopsy studies by Bates etal ${ }^{[13]}$ showed that pulmonary and extra-pulmonary tuberculosis was detected in over half of HIV-infected adults and four to five-folds more prevalent than in HIV-uninfected cases. Also, bacterial pneumonia was more prevalent in children than adults and was relatively unaffected by HIV status. The study also showed co-infections were common and correlation with premortem clinical diagnoses was low. Another study ${ }^{[14]}$ done at Muhimbili National Hospital, Dar es Salaam, Tanzaniarevealed high prevalence of undiagnosed tuberculosis during autopsy. For all study subjects who were found to have tuberculosis at autopsy, none had ever been diagnosed with tuberculosis before death. 


\section{Methods}

The aim of the study was determine the prevalence of lung lesions among medico-legal autopsies at a tertiary hospital.

A prospective cross sectional study was carried out at the Muhimbili National Hospital (MNH) department of pathology, to medico-legal autopsies received at the MNH Histopathology and Morbid Anatomy unit and the mortuary section. The study included 103 medico-legal autopsy cases over an eight month period (August 2015 - April 2016). Information on age, sex, marital status and occupation was collected from the first degree of the deceased, police and accident register report during autopsy. Data on gross features and weight of the lungs was collected during the postmortem and later the diagnosis was recorded after microscopic examinations of the obtained lung samples from each case.

Postmortem examination: To avoid changes in organ weight and structure, autopsy cases included were those performed within 48 hours after death. A thorough postmortem was done with particular attention to the gross examination of the lungs. In each case, the lungs and the heart were removed together. The lungs were thereafter weighed separately using YAMATO weighing balance. Samples from the right and left lobes were obtained in each case. The samples were fixed in $10 \%$ neutral buffered formalin and sent to the MNH Pathology laboratory for processing.

Sample processing and sectioning (Microtomy): Sections of $3 \mu \mathrm{m}$ were cut from paraffin blocks cooled on ice blocks using a microtome (SAKURA Model: SRM $200 \mathrm{CW}$ ). Sections were then floated on water bath at $45^{\circ} \mathrm{C}$ while mounted on standard frosted glass slides each labeled with a Postmortem (PM) number. Thereafter the slides were allowed to drain before being put on hot plate at $60^{\circ} \mathrm{C}$ for 15 minutes.

Staining Procedure: The sections were dewaxed in three changes of xylene three minutes each, and brought down to water through descending grades of ethyl alcohol (absolute: $95 \%, 80 \%$ and $70 \%$ respectively). The sections were then stained in Harris Hematoxylin for five minutes before being washed with tap water and differentiated in one percent Hydrochloric acid (HCL) in ethanol. The sections were again washed in running tap water and then blued for ten minutes. After being blued, the sections were counterstained in one percent aqueous eosin for five minutes before being washed in tap water and dehydrated in ascending grades of ethanol. The sections were then cleared in three changes of xylene then mounted in Destrine Plasticizer Xylene (DPX) and labeled accordingly.

Microscopic Examination: After the tissue samples were ready, they were examined under a light microscope and findings recorded in the data collecting sheet for each case.

\section{Statistical Analysis:}

Statistical analysis was performed using R statistical software (http://www.R-project.org/). Normality test assumption was measured using Shapiro-Wilk test. For continuous variables with normal distribution, 
Anova and TukeyHSD tests were used. Chi-Square test was employed for categorical variables, and Fisher's exact test applied for cell size less than 5. In addition, Local Polynomial Regression Fitting (loess) was used to assess the relationship between the total weight of the lungs and associated factors. This non-parametric regression analysis was used after the parametric regression normality and constant variance assumptions were violated.

P-value of $<0.05$ was considered to be statistically significant.

\section{Results}

\section{Age and Gender of the study subjects}

The study conducted gross and histopathology examination on 103 cases. Out of the 103 cases, $88.3 \%$ were males with mean age of $35.8+16.6$ years. The mean age for females was $31.6+10.4$ years (Table 1)

\section{Lung weights}

The mean weight of lungs for males was $625.7+138.5 \mathrm{~g}$ and $590.7+129.4 \mathrm{~g}$ for the right and left lungs, respectively. In female, the mean weight of lungs was slightly lower than males, weighing $616.7+166.1 \mathrm{~g}$ and $583.3+155.8 \mathrm{~g}$ for the right and left lung, respectively. (Table 2) However, group wise comparison found no statistical difference in mean weight of the right $(p=0.8359)$ and the left $(p=0.8613)$ lungs between male and female. In addition, the total weight of lungs was not related to gender. $(p=0.98642)$

Model assumption revealed trend between age and total lung weight. Local Polynomial Regression Fitting (loess) was run and showed the total lung weight to increase as one advances in age until 50 years. Thereafter, the total weight begins to decrease. (Figure 1)

\section{Lung lesions}

Histological examination found 55 (53.4\%) of the collected lung samples had some pathological microscopic findings. 51 (49.5\%) were from males. The leading pathological diagnosis was pulmonary congestion/oedema (22.3\%), followed by lobar pneumonia (18.5\%). Only one sample was found to have pulmonary tuberculosis. (Table 3 )

Histological diagnosis was found not to relate with gender. $(p=0.7734)$.

Figure 2 shows pulmonary emphysema to affect individuals above 60 years. Other lung lesions were found in individuals with age between 30 - 40 years. Anova analysis showed statistical significant difference between age and histological diagnosis. ( $P$-value $=0.0025)$ 
The total lung weight for most diseased lungs fall between 1200 - 1400 grams, except for the lung found to have pulmonary tuberculosis, which weighed below 1000 grams. (Figure 3) However, total weight of the lung was found not to relate to histological diagnosis.

\section{Discussion}

The sex and age distribution showed male predominance (88.3\%), with majority of the study subjects being on their early thirty's (Mean age of $35.8+16.6$ and $31.6+10.4$ years for male and female, respectively). The higher incidence of medico-legal deaths in male has also being found in other similar studies conducted in developing regions by Jhajj etal ${ }^{[10]}$, Selvam etal ${ }^{[11]}$ and Pathak etal ${ }^{[12]}$. This can in part be explained by the more evident rise of motorcycling as a prominent means of commercial transportation, engaging vast numbers of male youth and adults in the city. A significant number of the reported medico-legal deaths result from motorcycling-related accidents. Also, men in developing regions are the main breadwinners making them more vulnerable to exposure to environments that might lead to medico-legal deaths in their day-to-day undertakings, than women. The distribution may also reflect the like- hood of young men in indulging themselves in risk behaviors such as robbery, substance abuse and alcoholism than women.

\section{Lung weights}

The mean weight of the right and left lungs for males were similar to study by de la Grandmaison etal [8] in Caucasoid males in France. However, for females the current study recorded slightly higher mean weight. In addition, the mean weights for both lungs were higher than reported in studies done in India [5, 10,11,12] and Sub-Saharan Africa. ${ }^{\left[{ }^{[}\right]}$Lung weights have been shown to correlate to decedent's weight and height. ${ }^{[8,9]}$ However, this study did not compare the decedent's anthropometric measures with their corresponding lung weights. This could have aid in explaining and comparing the observed differences.

Even though the total mean lung weight was higher in males than females, as observed in other similar

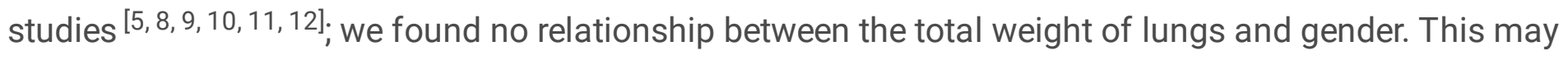
be attributed to few numbers of females in the current study.

Aging has been observed to be associated with reduction in mass of organs (especially the high metabolic rate organs) in the body. ${ }^{[15]}$ The model assumption showed some trend between age and total weight which was not captured by the linear regression (value of adjusted $R$ square was below 0.5 ). To capture this trend we ran local polynomial regression fitting (loess) between age and total weight. The results showed the total lung weight to increase as one advances in age, until 50 years. Thereafter, the total weight begins to decrease steadily. An autopsy study by Sawabe etal ${ }^{[16]}$ on standard organ weights showed a similar significant organ weight decrease in elderly Japanese (above 60 years) in both genders. 


\section{Lung lesions}

More than half $(53.4 \%)$ of the study subjects were found to have some pathological lung finding. This suggests high prevalence of undiagnosed lung pathologies in the general population. In part, this may be due to the fact that most of chronic lung diseases are not usually diagnosed until they are clinically apparent and moderately advanced. ${ }^{[17]}$ In addition, lack of surveillance systems and diagnostic tests required for the diagnosis and assessments of the severity of chronic respiratory diseases are not readily available. ${ }^{[7]}$

The leading pathological diagnosis in the current study was pulmonary congestion/oedema (22.3\%), followed by lobar pneumonia (18.5\%) and pneumoconiosis $(9.7 \%)$. These findings are similar to a study by Pathak etal ${ }^{[12]}$ on medico-legal autopsies in India, were the leading pathological diagnosis was pulmonary oedema $(37.8 \%)$ followed by pneumonic changes $(33 \%)$, even though the rates are higher in Pathak etal ${ }^{[12]}$ study.

Only one sample was found to have pulmonary tuberculosis. This is contrary to similar studies from other developing regions like India ${ }^{[10,11]}$ where tuberculosis was one the leading finding. Tuberculosis is still a major burden in Tanzania with prevalence of 295/100,000 [18], however, prevalence data in medicolegal autopsy cases is not available. A study by Kilale etal ${ }^{[14]}$ done at $\mathrm{MNH}$, Tanzania showed high prevalence of tuberculosis in clinical autopsies. The reason for high prevalence of undiagnosed tuberculosis cases with Kilale etal study can be due to selection of cases with chronic illnesses, and clinical presentation compatible with pulmonary pathology. Nevertheless, short study period may contribute to the current study's findings.

Fisher exact test showed no relation between histological diagnosis and gender. Again, this may be contributed to few female participants in the study.

To assess for the relationship between age and lung lesion, anova and Tukey HSD tests were run. From the anova test found statistical significant difference in mean age for different lung lesion ( $p$ value $=0.0025$ ). The main difference was between pulmonary emphysema and lobar pneumonia ( $p$ value $=0.0099)$ and pulmonary emphysema and pulmonary congestion/oedema ( $p$ value $=0.0113$ ). Pulmonary emphysema was observed to affect individuals above 60 years while lobar pneumonia and pulmonary congestion/oedema affected those between $30-40 \mathrm{yrs}$.

The total weight of the lungs was found not to relate to histological diagnosis. Since lung mass has been observed to correlate with subject's weight, height and BMI, direct intercomparison between subjects can be altered for lung mass changes brought by pathological disease process. ${ }^{[8,9]}$

\section{Limitations}


We were not able to capture lifestyle and other socio-economic factors that could affect the distribution of observed weight and lung pathologies. Also, the decedents' past medical history were not available. This study did not compare subjects' anthropometric measurements (weight, height and BMI) with their lung weights.

\section{Conclusion}

Males, especially youth and adults are the main victims of medicolegal deaths. The study has revealed high prevalence of undiagnosed lung diseases among victims of medicolegal deaths. Since these lung pathologies were incidental findings, not related to the cause of the deaths; the results may reflect the actual burden of lung diseases among the general population. Age has been found to relate to the observed histological diagnoses of the lungs.

\section{Abbreviations}

$\mathrm{BMI}$ - Body Mass Index

CRD - Chronic Respiratory Diseases

DPX - Destrine Plasticizer Xylene

HCL - Hydrochloric acid

HIV - Human Immune Deficiency Virus

HSD - Honest Significant Difference

MNH - Muhimbili National Hospital

MUHAS - Muhimbili University of Health and Allied Sciences

PM - Postmortem

WHO - World Health Organization

\section{Declarations}

\section{Ethical Considerations}

Approval to conduct the study was sought from Muhimbili University of Heath and Allied Sciences (MUHAS) ethical review board with reference number 2015-08-24/AEC/Vol.IX/414. Permission to collect data was issued by Muhimbili National hospital directorate of research. No direct subject identifiers were used in data collection. 


\section{Availability of data and materials}

The datasets used for analysis during the current study are available from the corresponding author on reasonable request

\section{Competing Interests}

The authors have no competing interests.

\section{Funding}

Efforts and all associated costs for the study were covered by Muhimbili University of Health and Allied Sciences, Muhimbili National Hospital and Authors.

\section{Author's contributions}

WFM conceived and designed the study. He also collected and analyzed the data, and wrote the paper. HAM participated in designing of the study, proposal development and data collection and analysis. UM participated in data analysis and wrote the manuscript.

All authors participated in critical review of final paper for submission.

\section{Acknowledgements}

We extend our sincere gratitude to all the staff of the pathology department and Central Pathology Laboratory (CPL) of MUHAS and MNH for unwavering support throughout data collection process and development of this paper. It is through their hard work, commitment and dedication to furtherance pathology research that we able to present this paper.

\section{References}

Vij T. Textbook of Forensic Medicine. New Delhi: Churchill Livingstone Pvt, 62; 2001.

Parikh CK.Parikh's Textbook of Medical Jurisprudence and Toxicology. 5th ed. Bombay: CBS Publishers and Distributors; 1990.

Dogra TD, Rudra A. Lyon's Medical Jurisprudence \&Toxicology. 11th ed. New Delhi: Delhi Law House. 2007. 
Kobzik L. The Lung. In: Cotran RS, Kumar V, Collins T. (eds.) Robbins Pathologic Basis of Disease.6th ed. Philadelphia, PA: WB Saunders; 1999. p697-701

Anil k, Aggarwal NK. Normal organ weights in Indian adults. Indmedica. 2006; 6(2)

World Health Organization. The global burden of disease: 2004 update. Geneva: WHO; 2008.

Bousquet J, Khaltaev N. (eds.) Global Surveillance, prevention and Control of respiratory diseases. A Comprehensive approach. Geneva: WHO, 2007.

la Grandmaison GL, Clairand I, Durigon M. Organ weight in 684 adult autopsies: new tables for a Caucasoid population. Forensic Sci Int. 2001; 119:149-54.

Mubbunu L, Bowa K, Petrenko V and Silitongo M. Correlation of internal organ weights with body weight and Body Height in Normal adult Zambians: A case study of Ndola Teaching Hospital. Anatomy Research International. 2018. Doi.org/10.1155/2018/4687538

Jhajj KK, Nibhoria S, Sandhu S, Bamra N, Padda P. A study of Histopathological Examination in Medicolegal Autopsies in Faridkot, Punjab, IJFMT 2013; 7(1): 76

Selvam V, Thamil R, Subramaniam PM,Vijayanath V. Prevalance of Common Diseases inLungs and Liver; A Histopathological study. JPBMS 2011; 12(09)

Pathak A, Mangal HM.Histo-Pathology examination in medico-legal autopsy-pros and cons. J Indian Forensic Sci. Apr-Jun 2010; 32(2):128-131.

Bates M, Mudenda V, Mwaba P, and Zumla A. Deaths due to respiratory tract infections in Africa: a review of autopsy studies. Curr Opin Pulm Med2013; 19: 229-237

Kilale etal.High prevalence of tuberculosis diagnosed during autopsy examination at Muhimbili National Hospital in Dar es Salaam, Tanzania.Tanzan J Health Res2013; 15(03)

St-Onge MP, Gallagher D. Body composition changes with aging: the cause or the result of alterations in metabolic rate and macronutrient oxidation? Nutrition. 2010;26(2):152-155. doi:10.1016/j.nut.2009.07.004

Sawabe, M. Saito, M. Naka, et al., Standard organ weights among elderly Japanese who died in hospital, including 50 centenarians, Pathol. Int. 56 (6) (2006) $315 \mathrm{e} 323$.

Celli BR, MacNee W, Agusti A, et al. Standards for the diagnosis and care of patients with chronic obstructive pulmonary disease (COPD) and asthma. Eur Respir J 2004; 23(6):932-946.

Ministry of Health and Social Welfare United Republic of Tanzania. Mid Term Review of the Health Sector Strategic Plan III 2009-2015, Main Report. Dar es Salaam: MOHSW; 2013. 
Figures

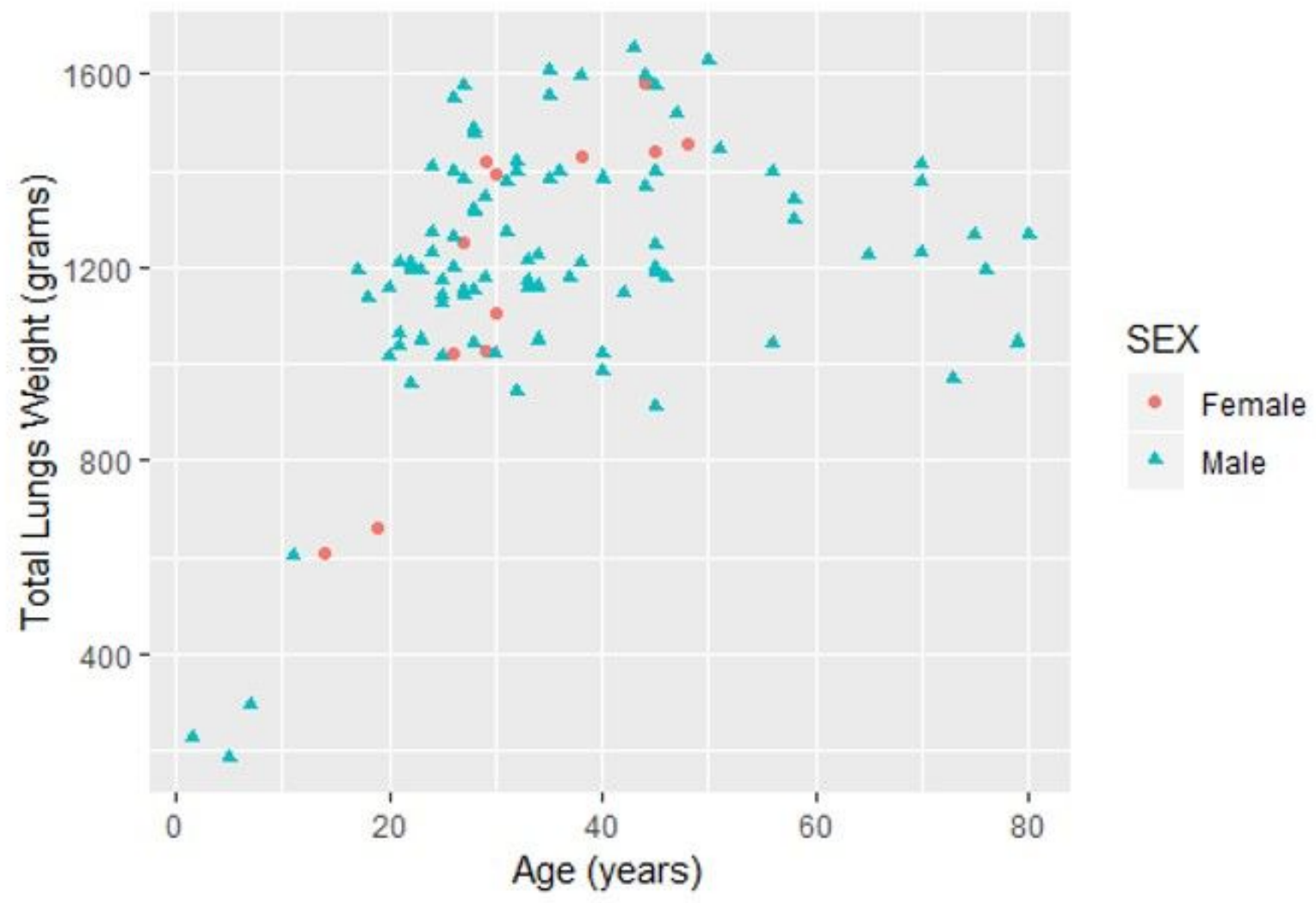

Figure 1: Pattern of total lung weight with age in male and female $(n=103)$

Figure 1 


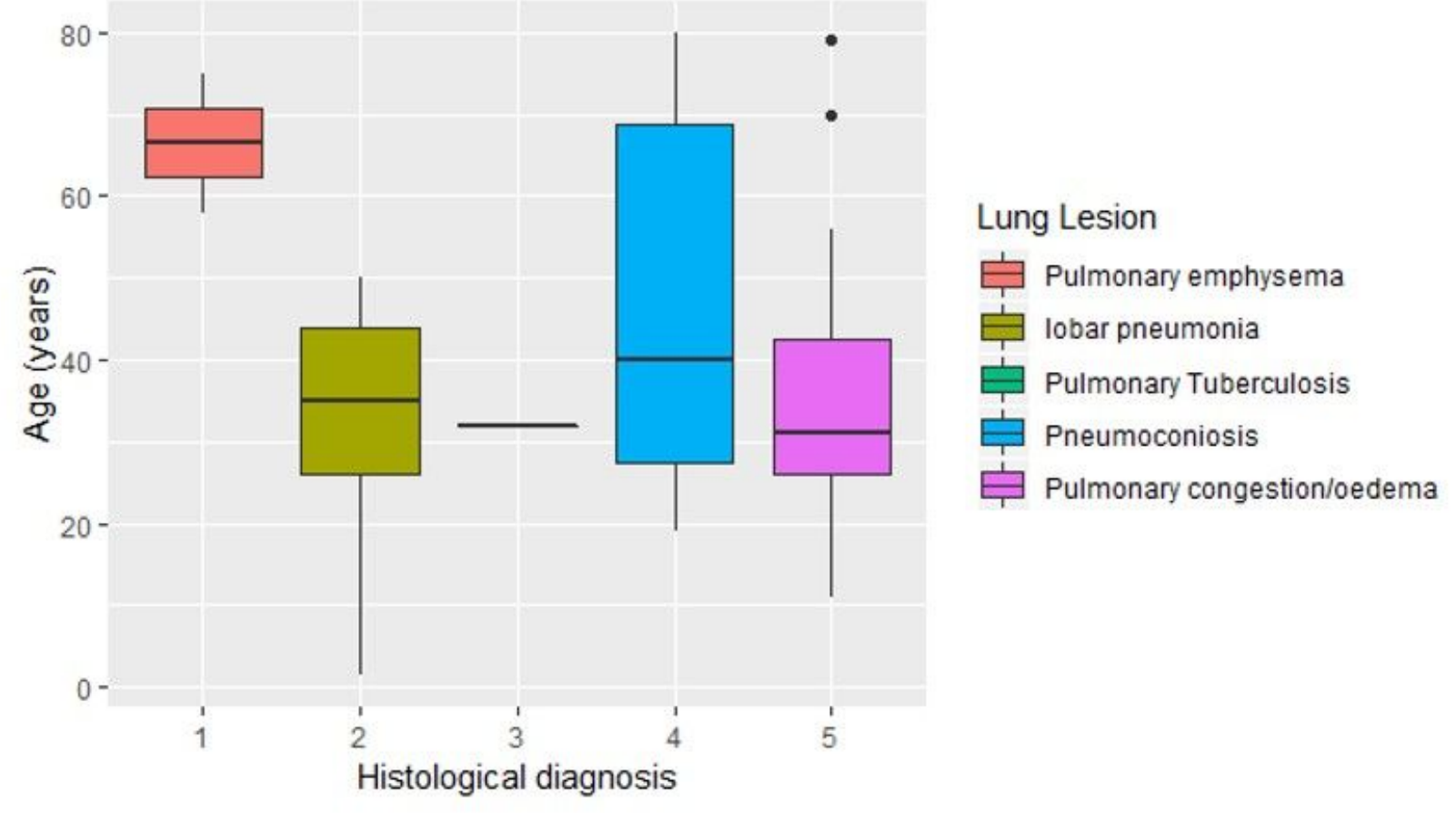

Figure 2: Distribution age with histological diagnosis $(\mathbf{n}=\mathbf{5 5})$

Figure 2

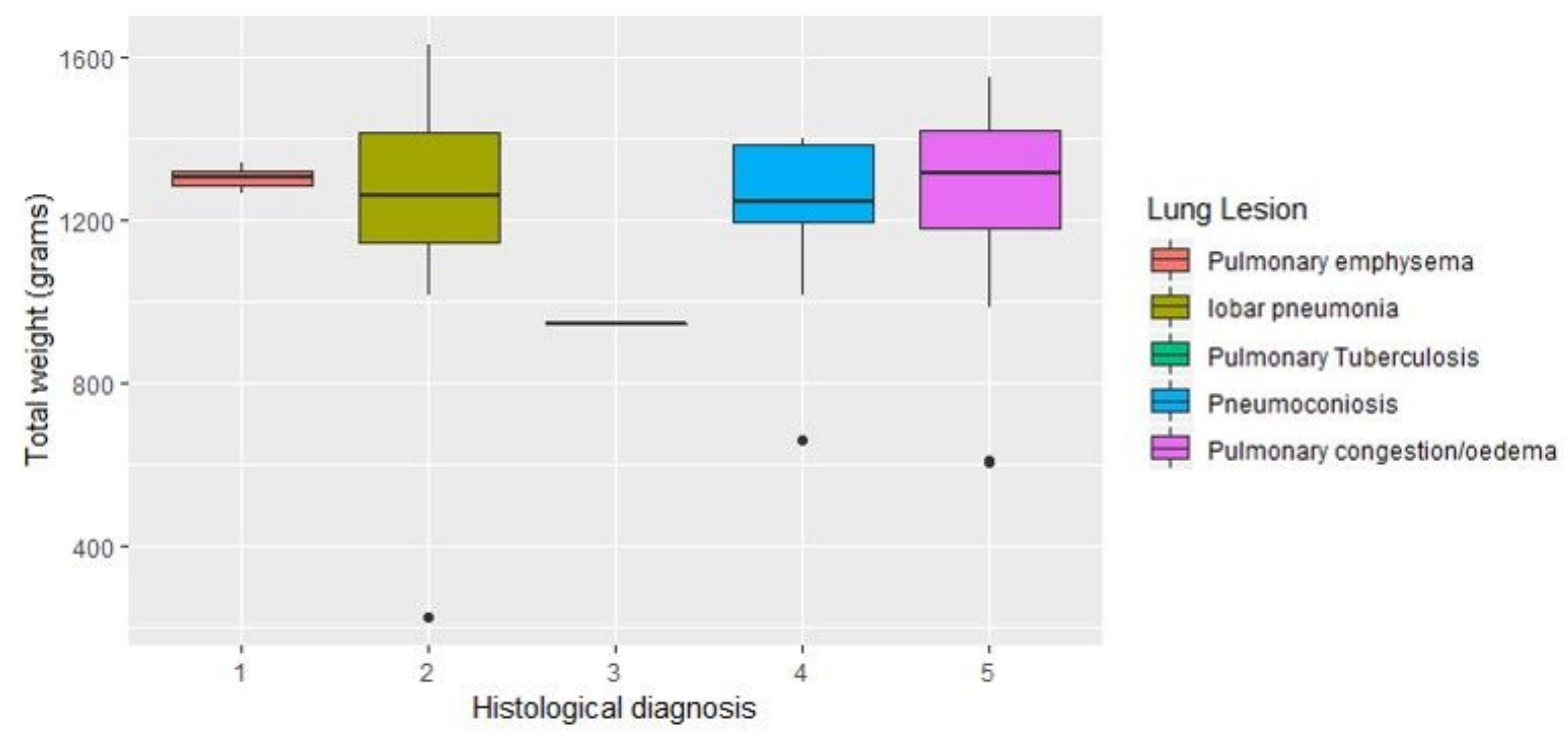

Figure 3: Distribution of total lung weight with histological diagnosis $(n=55)$ 


\section{Supplementary Files}

This is a list of supplementary files associated with this preprint. Click to download.

- Supplementaryfile.docx

- Tables.docx 